\title{
Residual costs in task switching: Testing the failure-to-engage hypothesis
}

\author{
SANDER NIEUWENHUIS \\ University of Amsterdam, Amsterdam, The Netherlands \\ and \\ STEPHEN MONSELL \\ University of Cambridge, Cambridge, England
}

\begin{abstract}
Reaction time is typically longer on trials on which the task changes. This switch cost is reduced by the opportunity to prepare for the change before the stimulus onset, but there remains a residual cost that resists reduction by further opportunity for preparation. De Jong (2000) proposed a model for evaluating the contribution to the residual cost of (1) failure to achieve endogenous task-set reconfiguration on a proportion of trials, and (2) limitations to the completeness of reconfiguration attainable by endogenous means. We report good fits of the model to the data from one previous and one new task-switching experiment, suggesting that the residual switch cost may indeed be attributable to a probabilistic failure to complete advance preparation. But strong incentives for preparation only marginally increased the estimated preparation probability, suggesting some intrinsic limitation to the ability to achieve endogenous preparation for a task switch on every trial.
\end{abstract}

Task switching has recently become a popular paradigm in the study of executive control. Participants are required to switch back and forth between two choice reaction time (RT) tasks afforded by the same class of stimulus. For example, participants might have to switch between classifying colored shapes by color or by shape, or between classifying digits as odd/even or as high/low. The task to be performed on a given trial can be determined either by a prespecified schedule (e.g., AABBAABB) or by an explicit cue presented prior to each stimulus. The responsestimulus interval (RSI) in the former case and the cuestimulus interval in the latter case can provide a preparation interval during which, on a switch trial, one might expect participants to attempt to reconfigure their cognitive processes for the changed task. The most basic observation is that the changing of tasks incurs a switch cost: Mean RT is longer (and error rate usually greater) when the task changes than when the same task is performed as on the previous trial.

Of particular interest here is the effect of the preparation interval on the switch cost. This is illustrated in Figure 1 (left panels) with data from Rogers and Monsell's (1995, Experiment 3) study, in which participants were presented on each trial with a character pair and had to respond manually in classifying either the digit (odd or even) or the

S.M. is now at the School of Psychology, University of Exeter, (e-mail: s.monsell@ex.ac.uk).We thank Ritske De Jong for providing sample programs for the application of the MMLM procedure and both him and Richard Ridderinkhof for helpful comments on an earlier draft of this paper. Correspondence concerning this article should be addressed to S. Nieuwenhuis, Department of Psychology, Green Hall, Princeton University, Princeton, NJ 08544 (e-mail: stn20@dds.nl). letter (vowel or consonant). The task was predictable according to an AABB task sequence and was also indicated by the position in which the character string was displayed (see the Method section). In different blocks, the RSI varied between 150 and $1,200 \mathrm{msec}$. As the preparation interval increased up to about half a second, there was a substantial reduction in switch cost from over $200 \mathrm{msec}$ to about half that value-a preparation effect. A further increase in the preparation interval did not further reduce the RT cost of a switch. We might say that there is a "residual" cost that is immune to elimination by the further lengthening of the preparation interval (see De Jong, 2000; Meiran, 1996; Rogers \& Monsell, 1995, for other examples) — even to as much as 2 or $3 \mathrm{sec}$ in some experiments.

The preparation effect, although unsurprising, is potentially important as an index of an endogenously triggered control process of task-set reconfiguration carried out by the participant prior to the stimulus onset. That the preparation effect indexes an active and optional process, rather than mere passive decay of interference from the previous task set, is demonstrated by the observation that the preparation effect can be prevented in conditions that should not prevent passive decay. For example, Rogers and Monsell (1995, Experiment 2) found no preparation effect when they varied the RSI randomly from trial to trial, and Goschke (2000) found that by articulating an irrelevant word during the preparation interval, the benefits of preparation were eliminated. Also, the preparation effect was observed in the cuing paradigm when the RSI was held constant but the cue-to-stimulus interval was varied (Meiran, 1996).

The residual cost seems more surprising. Why can participants not, given ample time to prepare, get as ready to perform the changed task as they had performed it on the 
previous trial-even where there is no repetition of stimulus or response? Three classes of explanation have been offered. According to Allport, Styles, and Hsieh's (1994) task-set inertia (TSI) theory, competing task sets vary in their degree of activation, which persists from one trial to the next. The residual switch cost is attributed to response selection on the postswitch trial's being prolonged by interference due to positive priming of the now-inappropriate task set and by negative priming due to the persistence of inhibition applied to the now-appropriate task set on the preswitch trial (Meuter \& Allport, 1999). (See Mayr \& Keele, 2000, for a variant of the idea of persisting task-set inhibition, and Allport \& Wylie, 2000; and Wylie \& Allport, 2000 , for a version of the TSI theory in which positive or negative priming is retrieved rather than being persistent.)

A second class of theory (Rogers \& Monsell, 1995; Rubinstein, Meyer, \& Evans, 2001) attributes the preparation effect and the residual cost to distinct components of the control process of task-set reconfiguration. As well as the endogenous component, which, as described above, can be carried out before the stimulus onset if the opportunity is available, Roger and Monsell posited an "exogenous" component - that is, one that requires the presence of the stimulus to initiate or complete and whose duration is responsible for the residual cost. Rubinstein et al. distinguished a goal-shifting process, which can occur before the stimulus, and a rule-activation process, which cannot. It is of course possible that both task-set inertia and a poststimulus control process contribute to the residual switch cost (Monsell, Yeung, \& Azuma, 2000).

Like the second class of theories, De Jong (2000) attributed the residual switch cost to the duration of a poststimulus control process. But De Jong (2000) made no distinction between endogenous and exogenous components of reconfiguration, instead positing a single preparation process, intention activation, that sometimes occurs before the stimulus and sometimes after. That is, he rejected the assumption that the residual cost arises from some intrinsic limitation to the achievement of asymptotic prestimulus readiness on every switch trial (whether due to task-set inertia or to the need for an exogenous control process, or both). Instead he claimed that, given a preparation interval, and the intention to prepare, participants succeed on only a proportion of the trials in completing the intention-activation process before the stimulus arrives, in which case they are in the prepared state, and performance is no different to that on a task repetition trial. On other trials, however, participants "fail to engage" intention activation before the stimulus onset and are in the unprepared state when it arrives, in which case intention activation must be accomplished after the onset to allow such task-specific processes as response selection to proceed, thus prolonging RTs. The residual cost in the mean $\mathrm{RT}$ is thus the result of a mixture, in some proportion, of prepared (no time cost) and unprepared switch trials (with a time cost due to poststimulus intention activation).
A mixture model of this kind makes predictions about RT distributions. To determine the relative contributions to the residual switch cost of a probabilistic failure to engage in advance preparation and other factors, De Jong (2000) described a formal two-state mixture model for the prepared switch trials and used two other conditions to provide empirical estimates of the RT distributions for the basis states that contribute to the mixture. In our case, the distribution of RTs from the nonswitch trials with a 1,200-msec RSI - the long/nonswitch condition - is taken to provide a reasonable empirical estimate of the prepared state. The distribution of RTs from the switch condition with a 150-msec RSI-the short/switch condition-served as the empirical estimate of the unprepared state, because in this condition, participants have little or no time to configure the system for the upcoming task. The model thus posits the following relation between the cumulative density function (CDF) for RT in the long/switch conditionthe alleged mixture distribution-and the CDFs for the two basis states:

$$
\begin{aligned}
F_{\text {long/switch }}(t)= & \alpha F_{\text {long/nonswitch }}(t-\delta) \\
& +(1-\alpha) F_{\text {short/switch }}(t),
\end{aligned}
$$

where $\alpha$ is the probability that advance reconfiguration is carried out during the long RSI-the preparation probability. $\delta$ is included to allow for the possible contribution to the residual switch cost of any influence that is approximately constant over switch trials-perhaps task-set inertia or the duration of an exogenous control process-whose effect is to shift the prepared CDF along the time axis. De Jong's (2000) failure-to-engage (FTE) hypothesis is that the zero-cost or reduced model, which is the special case of the above equation where $\delta=0 \mathrm{msec}$, is an adequate characterization of the residual switch costs found for young adults, with a variety of task pairs.

We computed the mixture model's fit to the correct RT data from Rogers and Monsell's (1995) Experiment 3, in which there was a mean residual time cost of $115 \mathrm{msec}$ (Figure 1, left panel). The basic procedure is, for each participant, to partition the RT distributions for the two basis conditions and the alleged mixture condition into five aligned bins. The multinomial maximum-likelihoodmethod (MMLM) of Yantis, Meyer, and Smith (1991) is then used to determine the proportion $\alpha$ with which RTs can be sampled from the two basis distributions (those for the switch/ short condition and those for the nonswitch/long condition) in order to maximize the goodness of fit with the observed proportions in the same five bins in the switch/long condition, by minimizing the likelihood-ratio statistic $G^{2}$, which is chi-square distributed for a valid reduced-mixture hypothesis $(\delta=0) .{ }^{1}$ Figure 2 (upper panel) shows the average, observed CDFs for the two basis conditions and the hypothesized mixture condition, together with the fit of the reduced-mixture model, as obtained by substituting the average estimate of $\alpha$ associated with the reduced model into the mixture model equation. It is clear that the reduced- 

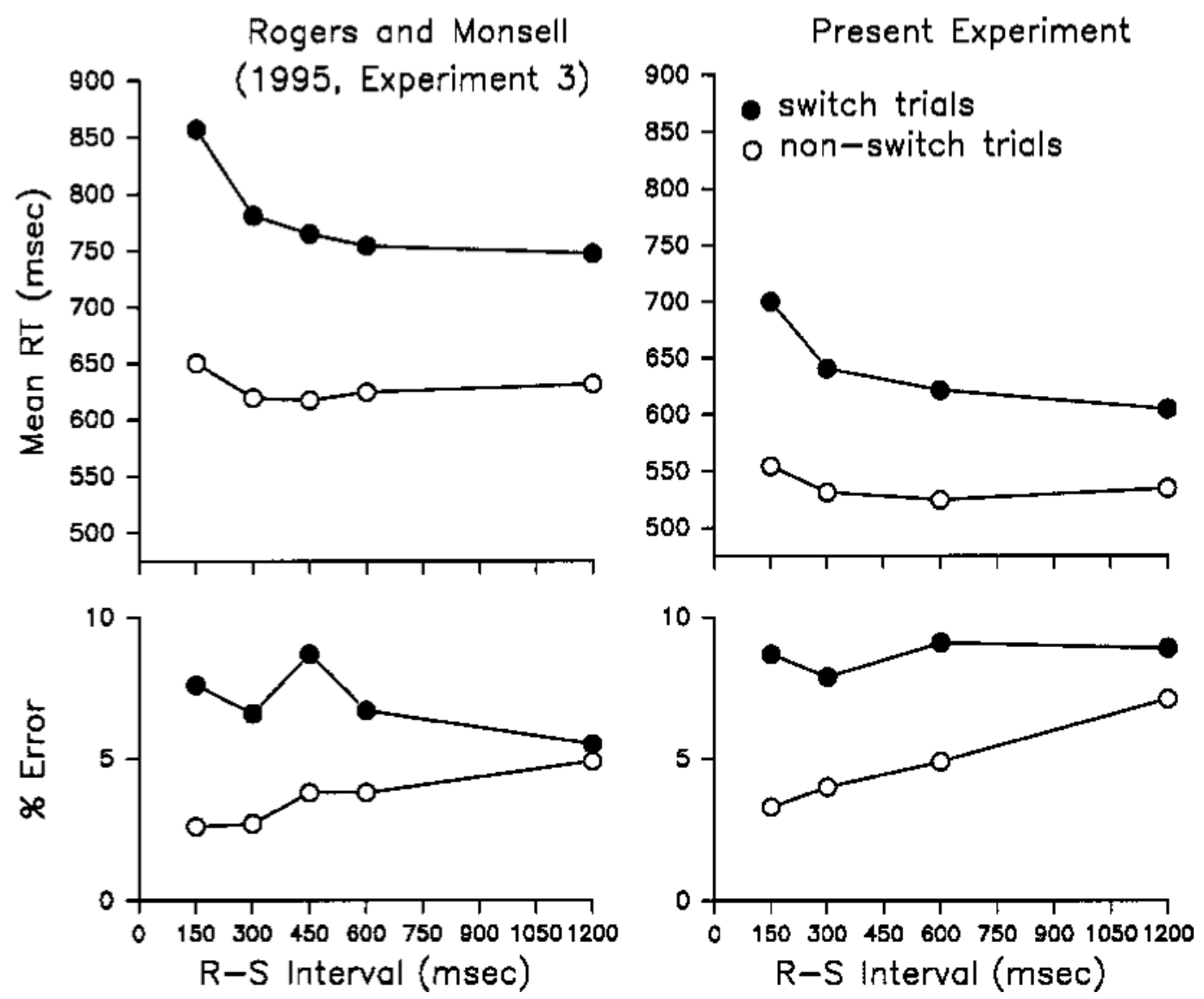

Figure 1. Mean correct reaction time and error rate as a function of trial type and RSI for Rogers and Monsell (1995, Experiment 3; left panels) and the present data (right panels).

mixture model gives a reasonably good approximation to the switch-1,200 CDF. The goodness-of-fit statistic indicates no significant difference between observed and fitted CDFs $\left[G^{2}(30)=29.4, p=.50\right]$. We cannot estimate the power of the test without specifying an alternative quantitativehypothesis. However, we can at least say that the full-mixture model, in which $\delta$ was allowed to vary freely, showed no significant improvement in fit compared with the reduced-mixture model $(p>.5): \delta$ had a mean of $-8 \mathrm{msec}(S E=12 \mathrm{msec})$. That is, we can confidently $(p<.05)$ reject a constant-duration poststimulus reconfiguration process longer than $20 \mathrm{msec}$, if coupled with a probabilistic poststimulus process on some switch trials. These modeling results suggest that the mean residual costs reported by Rogers and Monsell could, in principle, be attributed to probabilistic failures to engage in advance preparation. The mean estimated $\alpha$ was $.49(S E=.08)$, which would imply that the participants succeeded in advance preparation on only about half the switch trials at the longest RSI.

Let us for the moment take the good fit to indicate that the FTE hypothesis provides a correct account of the ori- gin of residual switch costs. What determines $\alpha$, the probability that a participant will succeed in effective intention activation before the stimulus? Why did the average participant in Rogers and Monsell's (1995) experiment do this on only half the trials? According to De Jong (2000), intention activation in advance of the stimulus is effortful. Participants will engage in it only if they both understand that it will reduce RT and wish to achieve that goal. Given such understanding, failures to engage should be less prevalent when response speed is assigned a high priority through incentive. Although Rogers and Monsell's participants were given standard RT instructions, were encouraged to prepare during the preparation interval, and were provided with end-of-block feedback on their mean RT, it is possible that either this incentive or their understanding of the benefits of preparation was insufficient. Furthermore, recent results (De Jong, 2000) indicate that the estimated preparation probability may increase as a function of the number of trials in a block, consistent with the idea that it is especially effortful to engage in advance preparation or to maintain the intention to do so over long sequences of trials. The aim of the present experi- 


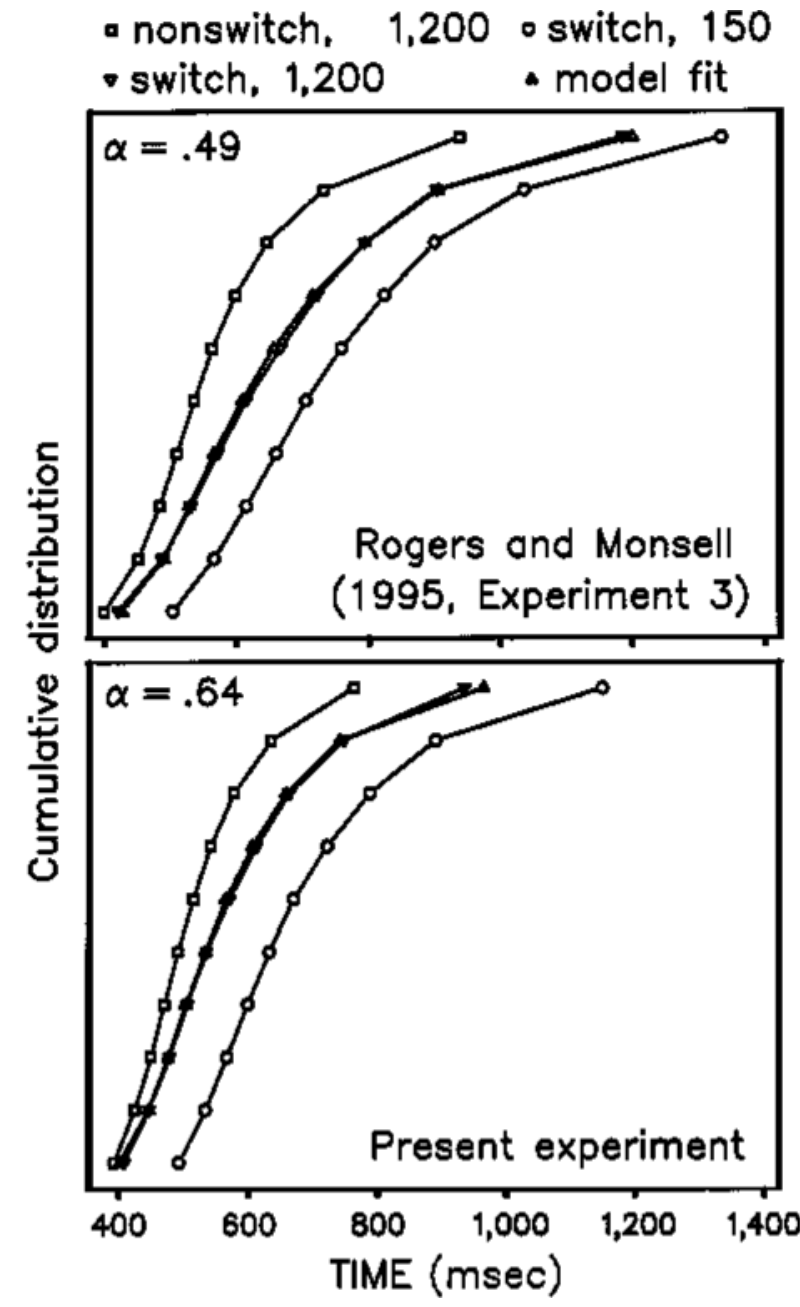

Figure 2. Empirical cumulative distribution functions for Rogers and Monsell (1995, Experiment 3; upper panel) and the present experiment (lower panel) for the short/switch, long/nonswitch, and long/switch conditions and the best-fitting CDF for the long/switch condition derived by using the average estimated mixture parameters associated with the reduced-mixture model.

ment was to try to raise the estimated preparation probability $\alpha$ to near unity and hence eliminate the residual switch costs by taking these considerations into account.

We therefore replicated the essential features of Rogers and Monsell's (1995) Experiment 3, in which the substantial residual cost afforded a considerable margin for reduction, but we made two crucial adjustments to the original design in order to emphasize to the participants the benefits of advance preparation and to ameliorate the effort involved. First, we used a payoff system in combination with extensive feedback to motivate the participants to the fullest to minimize RT. Pilot work established that this was effective in emphasizing speed over accuracy, thereby encouraging participants to make effective use of the longer preparation intervals. Second, although we used the same overall number of trials as Rogers and Monsell, we decreased the number in each block to 16 (it was 48 in
Rogers \& Monsell, 1995) in order to minimize fatigue-induced deterioration in effort from the beginning to the end of a block.

The FTE account suggests that maximizing our young and bright participants' motivation to push their RT performance to the limit, and minimizing cumulative fatigue, should substantially increase their estimated probability of preparation $\alpha$ and substantially reduce their mean residual switch costs, relative to Rogers and Monsell's (1995) participants (who were sampled from the same population). ${ }^{2}$ These predictions were further motivated by the finding, cited in De Jong (2000), of a strong negative correlation between single-task RT and $\alpha$ for individual participants.

\section{METHOD}

\section{Participants}

The participants were 12 students ( 7 women) from the University of Cambridge. They were paid $£ 6$ as a basic salary, plus a performance- related incentive bonus, as described below.

\section{Stimuli and Tasks}

Throughout each block, a 10-cm square divided into four quadrants was displayed on the computer screen. On each trial, a character pair was presented in a white uppercase Triplex font in the center of one quadrant. Each pair subtended a visual angle of $1.4^{\circ}$ both horizontally and vertically. The next stimulus was displayed clockwise in the next quadrant. One pair of adjacent display positions was assigned to the letter task and the other pair to the digit task, so that the display location served as a task cue, and the task changed predictably every second trial.

Depending on the task, the relevant character was either a letter or a digit. The second and irrelevant character was either a member of the other category, so that the response afforded by this character was either congruent or incongruent with the task-relevant response, or was drawn from a set of neutral characters. Consonants were sampled randomly from the set $<\mathrm{G}, \mathrm{K}, \mathrm{M}, \mathrm{R}\rangle$, vowels from the set $\langle\mathrm{A}, \mathrm{E}, \mathrm{I}, \mathrm{U}\rangle$, even digits from the set $\langle 2,4,6,8\rangle$, odd digits from the set $<3,5,7$, $9\rangle$, and neutral characters from the set $\langle \#, ?, *, \%\rangle$, with the restriction that a character could not be repeated on successive trials. The position of the task-relevant character within a pair was randomly determined on each trial. The participants responded with their left index finger (on the " $\mathrm{C}$ " key) to indicate "even" or "consonant" and their right index finger (on the "M" key) to indicate "odd" or "vowel."

\section{Design}

The participants were tested on 2 consecutive days. The first day started with 8 single-task training blocks of 24 trials for each task, with the irrelevant character always being neutral and an RSI of $150 \mathrm{msec}$. On both days, the participants received a practice set of 9 switch blocks, each with 16 trials and with a 150-msec RSI, before entering the experimental phase. This consisted of four sets of 15 blocks, one set for each RSI, each block consisting of 16 trials. RSI was held constant within each set. The order of the RSIs was counterbalanced across participants by means of a Latin square, with the order on the second day the reverse of that on the first. The irrelevant character was drawn from the neutral set on one third of the trials, and from the set associated with the congruent response on one third and with the incongruent response on the remaining third. Four warm-up trials at the beginning of each block were excluded from the data analyses. The remaining 12 experimental trials had one of each irrelevant character type (congruent, incongruent, or neutral) for each combination of task (letter, digit) and trial type (switch, nonswitch). 


\section{Procedure}

The RSI was $150,300,600$, or 1,200 msec and remained constant for a set. The stimulus was displayed until a response was registered. Detailed feedback was provided after each block in order to encourage the participants to strive continuously to improve their performance. Performance on each block completed that day was plotted on the screen with mean RT ( $T_{i}$ for the $i$ th block) on the abscissa, and number of errors $\left(E_{i}\right)$ on the ordinate, with the point for the block just completed ( $n$ ) highlighted. The diagonal, $\left(T_{c}-T_{n}\right) / 20-E_{n}=0$, was also displayed, where $T_{c}=$ mean RT for the last four blocks + $30 \mathrm{msec}$. The participants were encouraged to try constantly to "move their performance" toward the origin in order to keep it under the diagonal. After each block, a bonus, $\left(T_{c}-T_{n}\right) 20-E_{n}$ pence, was awarded (subject to a maximum of $5 \mathrm{p}$ and a minimum of $0 \mathrm{p}$ ). If the bonus was positive (i.e., performance fell under the diagonal), it was displayed on the screen accompanied by a musical tune, and the height of a cumulative "bonus meter" at the side of the display increased.

The participants were instructed to respond as fast as they could, while avoiding errors. After an error or a late response (RT $>$ $5,000 \mathrm{msec}$ ) a beep sounded for $1 \mathrm{sec}$, followed by another 1 -sec recovery period before the onset of the next stimulus. After a block in which more than two errors were made, the message "You have made too many errors. Please try to be more accurate" was displayed on the screen for $2 \mathrm{sec}$. The participants were also carefully instructed to use the RSI to get ready for the next task.

\section{RESULTS}

Trials with RTs of less than 200 msec were excluded from the analyses, as were trials immediately following an error. One participant from the present study was excluded from the analyses described below. (Unlike that for any other participant in the present study or in Rogers and Monsell's [1995], this participant's empirical "mixture" distribution was slower than the slow basis distribution-an anomaly for which neither the mixture model nor any other model of task switching or preparatory control can account.)

\section{RT and Errors}

The right panel of Figure 1 presents mean RTs and error rates as a function of trial type and RSI for the present experiment. The time costs of a task switch were reliably greater than zero $[F(1,10)=42.1, p<.001]$, as were the error costs $[F(1,10)=39.7, p<.001]$. The time costs decreased from $146 \mathrm{msec}$ at the shortest RSI to $69 \mathrm{msec}$ at the longest RSI. This effect of RSI was significant $[F(3,30)=$ $7.4, p<.005$ ], the simple effect of RSI on RT being reliable only for switch trials $[F(3,30)=6.1, p<.005]$. The residual time costs of $69 \mathrm{msec}(S E=10 \mathrm{msec})$ at RSI $=$ 1,200 were highly significant $(p<.001)$. The decrease of the error costs with RSI was not reliable $[F(3,30)=1.8$, $p=.19]$. The simple main effect of RSI on error rate was significant for nonswitch trials $[F(3,30)=5.9, p<.01]$, but not for switch trials $[F<1] .{ }^{3}$ Finally, the effects of task, practice (day of testing), and irrelevant character type were not appreciably different from those of Rogers and Monsell's (1995) Experiment 3.

\section{RT Distributions and Modeling Results}

Figure 2 (lower panel) presents the reduced-mixture model fit for the data from the present experiment. The obtained fit was again excellent $\left[G^{2}(33)=25.5, p=.68\right]$. Across participants, the mean estimate of $\alpha$ was now .64 $(S E=.04)$. The full-mixture model showed no significant improvement in fit compared with the reduced-mixture model $(p>.5)$. This finding was supported by a very small average estimate of $\delta(-6 \mathrm{msec}, S E=12 \mathrm{msec})$, which did not differ significantly from zero $(p>.5)$.

\section{Comparison with Rogers and Monsell (1995, Experiment 3)}

The average residual time costs in the present experiment were $69 \mathrm{msec}$, in comparison with $115 \mathrm{msec}$ in Rogers and Monsell. This difference just reached significance $[t(19)=1.7, p=.05]$. The average estimate of $\alpha$ in the present experiment was .64, in comparison with .49 in Rogers and Monsell's experiment. This difference also just reached significance $[t(19)=1.7, p=.05]$. However, the overall pattern in the present experiment was very similar to that of Rogers and Monsell, with both overall RT and switch costs being reduced.

\section{DISCUSSION}

The zero-cost or reduced-mixture model (De Jong, 2000) provides a good account of the data from the present experiment and from Rogers and Monsell's (1995) Experiment 3. Compared with the more parsimonious zero-cost model $(\delta=0)$, there was no significant improvement of fit of the full model, which incorporated a systematic (albeit constant) poststimulus component of reconfiguration, as represented by $\delta$. Thus, in line with the study of De Jong (2000), our results are compatible with the FTE hypothesis, suggesting that residual switch costs might be due to the participants' failure to engage in advance preparation despite ample opportunity to do so. Why intention-activation should have this probabilistic characteristic is unclear. Mayr and Kliegl (2000) have argued that an important and vulnerable component of task-set preparation is the retrieval of the relevant stimulus-response associations from long-term memory. According to this account, preparation failures reflect retrieval failures.

If the claim is correct that under the conditions explored here there is actually no residual cost on a substantial proportion of trials, this would seem troublesome for a taskset inertia account of the observed switch costs (like that of Allport et al., 1994) — unless of course it were amended so that positive or negative priming from a previously relevant task set became all or none and probabilistic. But this would constitute a marked departure from the conceptions of TSI that have prevailed hitherto. Perhaps Allport and Wylie's (2000) proposal that task sets and inhibition of task set are retrieved by stimuli with which they are associated could incorporate such an amendment. Alternatively, it might be argued that TSI contributes to switch costs only under limited conditions that are not in play here, such as switching between tasks of very unequal strength (see Monsell et al., 2000).

The apparently good fit to our data of the $\delta=0$ reduced model, along with the results of De Jong (2000) also chal- 
lenge the assumption of a task-set reconfiguration process with a poststimulus exogenous component (Rogers $\&$ Monsell, 1995) or rule-activation stage (Rubinstein et al., 2001) on every task-switch trial, at least under the present conditions. Exogenous driving cannot be essential to complete task-set reconfiguration if, on a substantial proportion of trials, performance on switch trials is as efficient as if there were no task switch. However, quantitative models of these and other theories of task switching need to be developed in order to examine whether they can provide alternative accounts of the specific properties of the RT distribution associated with the prepared switch condition. Furthermore, there is at least one published case (De Jong, 2001) in which, with older adults, the best-fitting model is one with a substantial $\delta(84 \mathrm{msec}$, with $\alpha=.33)$.

Taking Rogers and Monsell's (1995) Experiment 3 as a starting point, we sought in the present experiment to maximize the preparation probability and hence to substantially lower the residual cost by using strong incentives to minimize RT and short trial blocks to minimize the effort needed to sustain advance preparation over many trials. In one sense, these manipulations appear to have had the intended effect: Relative to Rogers and Monsell's experiment, mean RTs were reduced by more than $100 \mathrm{msec}$ without a concomitant increase in errors. Several participants commented that they felt very challenged by the bonus system and did everything they could to beat the criterion.

Notably, however, although these manipulations had effects on residual switch costs and $\alpha$ in the direction predicted by the FTE hypothesis, they did not eliminate the residual switch cost, which was still a robust $69 \mathrm{msec}$. The estimated mean preparation probability was only .15 greater than that estimated in Rogers and Monsell's (1995) experiment, and the spread of estimates does not suggest that some participants were successfully preparing all the time and others rarely. Thus, according to the FTE model, our carefully instructed, highly motivated, young, bright, and nonfatigued participants, were still failing to engage in intention activation on more than one third of the taskswitch trials. Hence, there does seem to be a more than merely motivational limitation on participants' ability to get themselves into the prepared state, albeit probabilistic rather than absolute. It is interesting to speculate what one could do to increase the probability of preparation further. One possibility is that the information in the cue is critical. For example, De Jong (2000) mentions obtaining a zero residual switch cost in a cuing experiment in which an explicit cue was used (e.g., the cue contained both red and blue when the next stimulus had to be classified red or blue). But this suggests that exogenous driving might be necessary to achieve intention activation in advance of the stimulus on $100 \%$ of the trials - an idea not so different from Rogers and Monsell's proposal of an exogenous substage of task-set reconfiguration! Further work is needed to explore this and other factors that may reduce the probability of failures to engage in advance preparation-if such failures are indeed implied by the good fit of the mixture model to our data.

\section{REFERENCES}

Allport, D. A., Styles, E. A., \& Hsieh, S. (1994). Shifting intentional set: Exploring the dynamic control of tasks. In C. Umiltà \& M. Moscovitsch (Eds.), Attention and performance XV: Conscious and nonconscious information processing (pp. 421-452). Cambridge, MA: MIT Press, Bradford Books.

Allport, [D.] A., \& Wylie, G. (2000). Task-switching, stimulusresponse bindings, and negative priming. In S. Monsell \& J. Driver (Eds.), Control of cognitive processes: Attention and performance XVIII (pp. 35-70). Cambridge, MA: MIT Press.

DE Jong, R. (2000). An intention-activation account of residual switch costs. In S. Monsell \& J. Driver (Eds.), Control of cognitive processes: Attention and performance XVIII (pp. 357-376). Cambridge, MA: MIT Press.

DE Jong, R. (2001). Adult age differences in goal activation and goal maintenance. European Journal of Cognitive Psychology, 13, 71-89.

GoschKe, T. (2000). Intentional reconfiguration and involuntary persistence in task-set switching. In S. Monsell \& J. Driver (Eds.), Control of cognitive processes: Attention and performance XVIII (pp. 331355). Cambridge, MA: MIT Press.

MaYr, U., \& KeELE, S. W. (2000). Changing internal constraints on action: The role of backward inhibition. Journal of Experimental Psychology: General, 129, 4-26.

Mayr, U., \& KLIEgL, R. (2000). Task-set switching and long-term memory retrieval. Journal of Experimental Psychology: Learning, Memory, \& Cognition, 26, 1124-1140.

Meiran, N. (1996). Reconfiguration of processing mode prior to task performance. Journal of Experimental Psychology: Learning, Memory, \& Cognition, 22, 1423-1442.

Meuter, R. F. I., \& Allport, [D.] A. (1999). Bilingual languageswitching and naming: Asymmetrical costs of language selection. Journal of Memory \& Language, 40, 25-40.

Monsell, S., Yeung, N., \& Azuma, R. (2000). Reconfiguration of task-set: Is it easier to switch to the weaker task? Psychological Research, 63, 250-264.

Rogers, R. D., \& Monsell, S. (1995). Costs of a predictable switch between simple cognitive tasks. Journal of Experimental Psychology: General, 124, 207-231.

Rubinstein, J. S., Meyer, D. E., \& Evans, J. E. (2001). Executive control of cognitive processes in task switching. Journal of Experimental Psychology: Human Perception \& Performance, 27, 763-797. Wylie, G., \& Allport, [D.] A. (2000). Task switching and the measurement of "switch costs." Psychological Research, 63, 212-233..

Yantis, S., Meyer, D. E., \& Smith, J. E. K. (1991). Analyses of multinomial mixture distributions: New tests for stochastic models of cognition and action. Psychological Bulletin, 110, 350-374.

\section{NOTES}

1. Each of the three empirical RT distributions used for this process was obtained by averaging the six CDFs for the combinations of day of testing ( 2 levels) $\times$ irrelevant character type (congruent, incongruent, neutral) for each participant in such a way as to exclude the systematic variance associated with these variables from the CDFs used in the test of the mixture model. Ideally, we would estimate and average separate CDFs for other possible subcategorizations known to affect RT and/or switch costs, such as response repetition. However, in order to obtain adequate estimates of CDFs, it is necessary to pool over at least 25 trials. Response repetition has a smaller effect than congruence or day, and although it interacts with switch costs, there is relatively little interaction between response repetition and RSI on switch trials (Rogers \& Monsell, 1995, Figure 6). Only in the presence of a systematic interaction of this type would the pooling procedure compromise the mixture model fit. In other respects, including the choice of bins, we followed exactly the procedure of De Jong (2000).

2. A within-experiments comparison would have been ideal. However, given an effective replication of Rogers and Monsell's (1995) conditions and selection of participants from the same population, this comes close. We note that a within-subjects contrast of incentive and nonincentive conditions would not be desirable because of the likelihood of asymmetrical transfer effects. 
3. The stability of the nonswitch RT over RSI is important for the logic of using the short/switch and long/nonswitch conditions as the basis distributions. However, the increase in error rate with RSI on nonswitch trials might suggest an RSI effect that compromises this logic. There seem to be three possibilities. (1) This trend reflects a process restricted to error generation (e.g., occasional "lapses" of concentration); the logic of any test applied to RT distributions is unaffected. (2) The mechanism of this RSI effect applies both to nonswitch trials and to prepared switch trials, in which case the logic of the mixture test is unaffected. (3) A long RSI causes a loss of arousal or readiness on the nonswitch trials only (because the participant has nothing to do but "maintain" the previous task set), but there is also a compensating shift of speed-accuracy tradeoff that counteracts the effect of RSI on RT. Since these two effects cancel out to leave at least the mean of the RT distribution where it ought to be, there is little impact on the logic of the mixture test. Moreover, there is a similar (though admittedly less pronounced) RSI effect on the nonswitch error rate in Rogers and Monsell's (1995) data (Figure 1, bottom left panel). Thus, even if this effect reflects something that causes both experiments slightly to misestimate $\alpha$, the important effect of experiment on $\alpha$ should remain relatively unaffected.

(Manuscript received August 15, 2000; revision accepted for publication April 25, 2001.)

\section{SCiP 2002 \\ Call for Papers}

The 32nd annual meeting of the Society for Computers in Psychology will be held at the Hyatt hotel (Kansas City, MO) on November 21, 2002, before the opening of the annual meeting of the Psychonomic Society. Typically, the program includes posters, papers, symposia, and tutorials dealing with the use of computers in all areas of psychology, including research, education, and industry. Sessions will provide an opportunity for experienced computer users to exchange information on various aspects of computing and for less experienced users to attend tutorials. In addition, there will be invited addresses of general interest and commercial exhibits. Instructions for submitting papers and examples of sessions at recent conferences can be found at the SCiP website: http://www.lafayette.edu/allanr/scip.html.

Specific topics suggested for this year's conference include, but are in no way limited to, research and teaching on the Web, modeling of cognitive processes, and computer-based tools for research. As usual, articles based on papers presented at the SCiP meeting will be invited for an issue of Behavior Research Methods, Instruments, \& Computers that focuses on the conference.

You need not be a member of SCiP to present at the conference (although you are certainly encouraged to join). Presentations have traditionally been talks of 15 to 20 minutes. Symposia are groups of about 4 to 6 talks on a related topic. If you are interested in organizing a symposium, or have a suggestion for a symposium that you would like to see organized, please contact the program chair as early as possible. If you are interested in presenting a talk or poster, you must submit your materials by the deadline of June 28th, 2002 .

If computers have become integral to your work as a psychologist-or if you'd like to learn more about using technology to improve your instruction, research, communication, and analyses-come to Kansas City and join us for a stimulating day of presentations, demonstrations, and tutorials!

You are encouraged to contact Robert W. Proctor (program chair) with questions or comments.

Address: Department of Psychological Sciences, Purdue University, West Lafayette, IN 47907-1364.

E-mail: proctor@psych.purdue.edu. 